\title{
Manifestation of polaronic effects in Josephson currents
}

\author{
A.V. Parafilo ${ }^{1}$, I.V. Krive ${ }^{1,2,3}$, R.I. Shekhter ${ }^{2}$, Y.W. Park ${ }^{4}$, and M. Jonson ${ }^{2,5,6}$ \\ ${ }^{1}$ B. Verkin Institute for Low Temperature Physics and Engineering of the National Academy of Sciences of Ukraine \\ 47 Lenin Ave., Kharkov 61103, Ukraine \\ E-mail: parafilo_sand@mail.ru \\ ${ }^{2}$ Department of Physics, University of Gothenburg, SE-412 96 Göteborg, Sweden \\ ${ }^{3}$ Physical Department, V.N. Karazin National University, Kharkov 61077, Ukraine \\ ${ }^{4}$ Department of Physics and Astronomy, Seoul National University, 599 Gwanak-ro, Gwanak-gu, Seoul 151-747, Korea \\ ${ }^{5}$ SUPA, Institute of Photonics and Quantum Sciences \\ Heriot-Watt University, Edinburgh EH14 4AS, Scotland, UK \\ ${ }^{6}$ Department of Physics, Division of Quantum Phases and Devices, Konkuk University, Seoul 143-701, Korea
}

Received April 24, 2013

\begin{abstract}
Polaronic effects on the Josephson current through a vibrating quantum dot are considered. In the regime of strong electron-vibron interactions they lead to a power-law suppression of the critical current. This is manifested in an anomalous temperature dependence of the critical current at temperatures of the order of the polaronic energy shift.
\end{abstract}

PACS: 74.45.+c Proximity effects; Andreev effect; SN and SNS junctions;

85.85.+j Micro- and nano-electromechanical systems (MEMS/NEMS) and devices;

71.38.-k Polarons and electron-phonon interactions;

74.78.Na Mesoscopic and nanoscale systems.

Keywords: electron-vibron interactions, Josephson current, polaronic effects, Franck-Condon blockade.

\section{Introduction}

Vibrational effects strongly influence electron transport in molecular transistors (see, e.g., the Rev. 1). As a rule, strong electron-vibron interactions in quantum dots (QD) suppress the electrical current at low temperatures and small bias voltages. This phenomenon is known as the Franck-Condon blockade [2]. It is manifested in a step-like dependence of the current on bias voltage (signalling the successive opening of additional inelastic channels for electron transport) and in an anomalous temperature dependence of the conductance caused by the appearance of an additional low-energy scale (the vibron energy $\hbar \omega_{0}$ ) of importance for electron transport. Recently these theoretical predictions (see, e.g., the Rev. 3) were observed in electron tunneling through suspended single-wall carbon nanotubes [4] and in carbon nanopeapod-based molecular transistors [5].

Another effect of electron-vibron interactions in quantum dots is single-electron "shuttling" [6] (see also the Rev. 7). Shuttling occurs when electron tunneling ampli- tudes are sensitive to the displacement of a quantum dot located in the gap between source- and drain electrodes. Electron shuttling is a nonequilibrium phenomenon, which strongly enhances the electrical current at large bias voltages, $e V \gg \hbar \omega_{0}$. Therefore, depending on the physical situation the electromechanical coupling in quantum dots can in principle either suppress (polaronic effect) or enhance (shuttling effect) the electrical current.

For more than a decade single-wall carbon nanotubes (SWNTs) have been used as normal elements (quantum dots) in Josephson junctions (see, e.g., Refs. 8 and 9). Although suspended nanotubes were utilized as weak links in superconducting devices [10], the influence of vibrational effects on supercurrent so far was not observe in experiment [11]. The interplay of proximity-induced superconductivity and mechanical vibrations in nanotube-based SNS junctions has been investigated in several theoretical papers [13-16] (see also the brief Rev. 17). The influence of vibrations on a nonresonant Josephson current through a single-level quantum dot was first considered in Ref. 13, where it was shown that in the regime of "hard" vibrons $\hbar \omega_{0} \gtrsim \Delta_{0} \quad\left(\Delta_{0}\right.$ is the 
superconducting gap) the supercurrent is suppressed due to a strong (exponential) narrowing of the partial level widths $\Gamma_{j}=\Gamma_{0 j} \exp \left(-g^{2}\right)(j=L / R$ stands for "left" and "right" partial width, $g$ is the dimensionless strength of electronvibron interaction). Notice that an exponential suppression (Franck-Condon blockade [2]) of the critical current $J_{c} \propto \Gamma_{L} \Gamma_{R} \exp \left(-2 g^{2}\right)$ takes place only in the limit $\hbar \omega_{0} \gtrsim \Delta_{0}$ [13]. In this case the vibrational side-band levels $\left(\varepsilon_{0}+n \hbar \omega_{0}\right.$, where $n$ is an integer) fall within the continuum spectrum and therefore only weakly influence the dc Josephson current in a short $\left(L \ll \xi_{0}=\hbar v_{F} / \Delta_{0}\right)$ junction. The exponential suppression of the elastic $(n=0)$ channel is nothing but a manifestation of the Franck-Condon blockade in the Josephson current.

In this work we will show that in the opposite limit, where $\hbar \omega_{0} \ll \Delta_{0}$, the virtual population of vibrational side-band levels in the superconducting gap lifts the Franck-Condon blockade. For strong electron-vibron interactions we find that this results in a weaker, power-lawlike suppression of the critical Josephson current.

The influence of "soft" vibrons, $\hbar \omega_{0} \ll \Delta_{0}$, on the Josephson current was studied in Ref. 15. In this case the vibrational subsystem can be treated quasiclassically and the corresponding effective potential energy for vibrons, induced by "polaronic effects" on the Andreev levels, becomes anharmonic with minima that depend on the superconducting phase difference $\varphi$. Numerical calculations performed in Ref. 15 in the regime $\Gamma_{0} \gtrsim \Delta_{0}$ revealed a vibron-induced supression of the critical current and a weak influence of the electromechanical coupling on the current-phase relation. An analogous result concerning the Josephson current was obtained in Ref. 16 for the whole region of vibron frequencies and weak electron-vibron interaction (in Ref. 16 quantized electromagnetic fluctuations in the superconducting circuit play the role of the vibrons in our terminology).

The purpose of our paper is to predict experimental signatures of strong electron-vibron interactions (polaronic effects) in Josephson junctions. The suppression of the critical current by itself does not provide an experimental test for vibrational effects. This is because the multiplicative renormalization of level widths is hard to detect (since one would have to be able to vary the electron-vibron interaction strength in a single experiment).

We consider a simple model of an S-QD-S junction characterized by four independent parameters: the angular frequency $\omega_{0}$ of the vibrational mode (only one vibrational QD mode is considered), the energy $\varepsilon_{0}$ and the width $\Gamma_{0}$ of a single QD electron level, and the characteristic electron-vibron interaction energy $\lambda$ (all these energies are assumed to be much smaller then the superconducting gap $\Delta_{0}$ ). In the quasiclassical limit $\omega_{0} \rightarrow 0$ this model can easily be solved analytically. In the strong interaction region $\lambda \gg \hbar \omega_{0}$ we find a power-law-like suppression of the critical current, $J_{c} \propto \lambda^{-2}$, which is due to a displace-

ment of the QD accompanied by a polaronic shift $\varepsilon_{p} \simeq \lambda^{2} / \hbar \omega_{0} \gg \varepsilon_{0}$ of the electron energy level $\varepsilon_{0}$. At low temperatures $T \ll \hbar \omega_{0}$ the manifestation of polaronic effects in the Josephson current is the appearance of cusps [15] in the current-phase relation $\left(\lambda^{2} \simeq \hbar \omega_{0} \Gamma_{0}\right)$ and a hysteretic behavior of the supercurrent as a function of an increasing/decreasing value of the superconducting phase difference $\varphi$ or of a gate voltage $V_{g}$.

The most convincing manifestation of polaronic effects in the Josephson current is the anomalous temperature dependence of the critical current. Temperature effects (caused by thermally excited vibrons) tend to restore the symmetric (unshifted) state of the QD and therefore increase the supercurrent up to some "critical" temperature $T_{m}$ of the order of the polaronic energy, $T_{m} \simeq \lambda^{2} / \hbar \omega_{0}$. Here, an abrupt transition to the standard temperature dependence of the critical current, $J_{c} \propto \tanh \left(\varepsilon_{0} / 2 T\right)$, occurs. In the quasiclassical approximation this transition takes the form of a sudden jump in the current. In the strong coupling regime the height of the jump is of the order of the resonant current and is not sensitive to other model parameters. We have also been able to show that a similar effect takes place in a model, where the tunneling matrix elements strongly depend on the QD position (as happens when the QD vibrates parallel to rather than perpendicular to an imaginary line between the source and drain electrodes, see Figs. 1(a) and 1(b)). Therefore the described anomalous temperature dependence of the critical current is indeed a signature of polaronic effects on the supercurrent through a Josephson junction containing a soft $\left(\hbar \omega_{0} \ll \Delta_{0}\right)$ vibrational subsystem.

In the quasiclassical approach the behavior of the critical current in the vicinity of the polaronic temperature $T_{m}$ is not controlled by the approximation. In order to verify the predicted anomalous temperature dependence of criti-

(a)

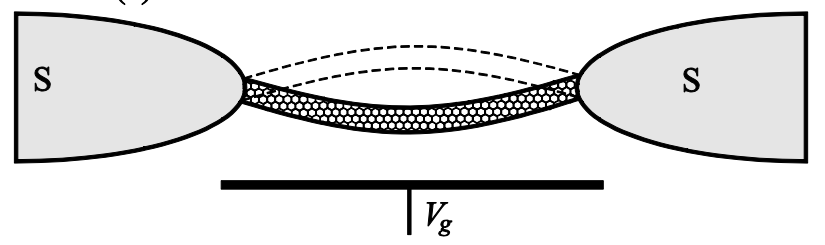

(b)

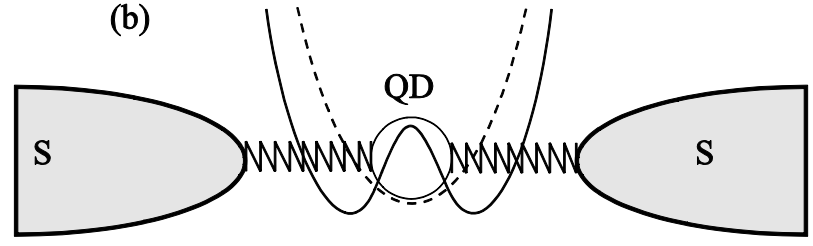

Fig. 1. Two examples of a system where a movable quantum dot serves as a weak link between two superconducting electrodes: the weak link is a suspended carbon nanotube electrostatically interacting with a gate electrode $\left(V_{g}\right)(\mathrm{a})$, the weak link is a fullerene molecule confined by an effective potential in the gap between source and drain electrodes (b). 
cal current we have therefore also calculated the Josephson current through a vibrating quantum dot by a Green's function method. In Ref. 18 a general formula for the supercurrent through an interacting quantum dot was suggested. The proposed expression generalizes the well-known Meir-Wingreen formula [19] to the case of superconducting leads and allows one to express the supercurrent in terms of the retarded QD Green's function in the Nambu representation. This can be evaluated analytically in the so called polaron tunneling approximation [20,21], which is valid when the characteristic polaron energy $\varepsilon_{p} \simeq \lambda^{2} / \hbar \omega_{0}$ is much larger than the level width $\Gamma_{0}$, i.e., when $\lambda^{2} \gg \hbar \omega_{0} \Gamma_{0}$. This inequality coincides with the one used to define the validity of the quasiclassical approach and allows us to cross-check our analytical results for the critical current at $T=0$ and to obtain the temperature dependence of the critical current in a quantum mechanical treatment of the problem. At low temperatures, $T \ll \hbar \omega_{0}$, we find a result for the critical current that exactly coincides with the one we obtained in the quasiclassical approximation. A numerical calculation of $J_{c}(T)$ confirmed our assertion that polaronic effects in Josephson current are manifested in an anomalous temperature dependence of the critical current.

\section{Polaronic suppression of critical current. Quasiclassical approach}

Following Refs. 13 and 15 we consider the simplest model that can describe vibrational effects in the Josephson current. The model describes a movable (vibrating) quantum dot with a single spin-degenerate electron level $\left(\varepsilon_{0}\right)$ weakly coupled to bulk superconductors (see Fig. 1). The QD Hamiltonian takes the form

$$
\begin{gathered}
H_{Q D}=\sum_{\sigma=\uparrow, \downarrow} \varepsilon_{0} d_{\sigma}^{\dagger} d_{\sigma}+\frac{\lambda}{\sqrt{2}}\left(\hat{n}_{\uparrow}+\hat{n}_{\downarrow}\right)\left(b^{\dagger}+b\right)+ \\
+\hbar \omega_{0} b^{\dagger} b+U \hat{n}_{\uparrow} \hat{n}_{\downarrow},
\end{gathered}
$$

where $\varepsilon_{0}$ is the energy of the dot level measured from the Fermi level, $d_{\sigma}^{\dagger}\left(d_{\sigma}\right)$ is the electron creation (destruction) operator, $\hat{n}_{\sigma}=d_{\sigma}^{\dagger} d_{\sigma}, \lambda$ is the electron-vibron interaction energy, $b^{\dagger}(b)$ is the vibron creation (destruction) operator, $\omega_{0}$ is the vibron frequency, and $U$ is the Coulomb energy. In this section we will not consider electron-electron interaction effects, assuming that $\lambda \gg U$.

The superconducting left $(j=L)$ and right $(j=R)$ electrodes are described by the standard BCS Hamiltonian

$$
H_{j}=\sum_{k, \sigma} \varepsilon_{k j} c_{j, k \sigma}^{\dagger} c_{j, k \sigma}-\left(\sum_{k} \Delta_{j} c_{j, k \uparrow}^{\dagger} c_{j,-k \downarrow}^{\dagger}+\text { h.c. }\right)
$$

$\left(\Delta_{j}=\Delta_{0} \mathrm{e}^{i \varphi_{j}}\right.$ is the superconducting order parameter). The coupling of the QD to the leads is modeled by the tunneling Hamiltonian

$$
H_{t}^{(j)}=\sum_{k, \sigma} t_{k j} c_{j, k \sigma}^{\dagger} d_{\sigma}+\text { h.c. }
$$

In what follows we will assume the tunneling amplitudes $t_{k j}$ to be independent of energy, $t_{k j} \simeq t_{0 j}$. This assumption is justified when all characteristic energies in our problem $\varepsilon_{0}, \lambda, \hbar \omega_{0}, \Gamma_{0}, T \quad\left(\Gamma_{0}=2 \pi \nu\left(\varepsilon_{F}\right)\left|t_{0 j}\right|^{2}\right.$ is the level width, $T$ is the temperature, $v$ is the density of states) are much smaller then $\Delta_{0}$. Additionally, in this Section the tunneling amplitudes are considered to be coordinate-independent. This is the case, e.g., for a suspended single-wall carbon nanotube subject to a gate potential as sketched in Fig. 1(a), from which it is clear that nanotube vibrations do not affect the coupling of electronic states in the tube and in the leads. The opposite case of a strong dependence of the tunneling matrix elements on the QD coordinate $\hat{x}=x_{0}\left(b^{\dagger}+b\right) / \sqrt{2} \quad\left(x_{0}\right.$ is the amplitude of zero-point fluctuations), see Fig. 1(b), will be studied in Sec. 4.

The influence of a vibrational degree of freedom on the supercurrent is different for "hard" ( $\hbar \omega_{0} \gtrsim \Delta_{0}$ ) and "soft" ( $\left.\hbar \omega_{0} \ll \Delta_{0}\right)$ vibrons. In the first case the vibrational subsystem is always in its vacuum (unshifted) state $\langle\hat{x}\rangle=0$ and zero-point fluctuations of the QD coordinate results in a Franck-Condon blockade of the critical current [13]. Soft vibrons, on the other hand, by means of interacting with the Andreev bound states (see below) are ready to form a new ground state $\langle\hat{x}\rangle \neq 0$ in order to minimize the total energy. Such a transition mimics the formation of a gap in the conduction band of electrons and the condensation of phonons that follow from a Peierls phase transition.

In the limit $\omega_{0} \rightarrow 0$ the vibrational subsystem can be treated quasiclassically. When $\left|\varepsilon_{0}\right|, \Gamma_{0} \ll \Delta_{0}$ only subgap states contribute to the Josephson current. Replacing the dimensionless coordinate $\hat{x}_{c}=\hat{x} / x_{0}=\left(b^{\dagger}+b\right) / \sqrt{2}$ in Eq. (1) by the classical variable $x_{c} \gg 1$, it is easy to find the spectrum of the two Andreev bound states in our problem as $E_{A}^{ \pm}= \pm E_{A}$, with

$$
E_{A}=\sqrt{\left(\varepsilon_{0}+\lambda x_{c}\right)^{2}+\frac{1}{4}\left(\Gamma_{L}^{2}+\Gamma_{R}^{2}+2 \Gamma_{L} \Gamma_{R} \cos \varphi\right)} .
$$

At zero temperature (or more precisely if $T \ll \hbar \omega_{0}$ ) the Josephson current is given by the derivative of the ground state energy $E_{0}$ of the weak link with respect to the superconducting phase difference $\varphi$,

$$
J(\varphi)=\frac{2 e}{\hbar} \frac{\partial E_{0}}{\partial \varphi},
$$

where in our case $E_{0}\left(\varphi, x_{c}(\varphi)\right)$ depends on the vibrational degree of freedom $x_{c}(\varphi)$. The equilibrium coordinate $x_{c}(\varphi)$ can be found by minimizing the total energy,

$$
\frac{\partial E_{0}\left(\varphi, x_{c}\right)}{\partial x_{c}}=0, \quad E_{0}\left(\varphi, x_{c}\right)=\frac{\hbar \omega_{0}}{2} x_{c}^{2}-E_{A}\left(\varphi, x_{c}\right) .
$$


First we analyze the case of resonant tunneling, $\varepsilon_{0}=0$. Recall that in the absence of electron-vibron interaction $(\lambda=0)$ the expression for the resonant Josephson current through a symmetric $\left(\Gamma_{L}=\Gamma_{R}=\Gamma_{0}\right)$ junction is (see, e.g., $[23,24])$

$$
J_{r}(\varphi)=\frac{e \Gamma_{0}}{\hbar} \sin \left(\frac{\varphi}{2}\right) \operatorname{sgn}\left(\cos \left(\frac{\varphi}{2}\right)\right) .
$$

In a strongly asymmetric junction, $\Gamma_{L(R)} \gg \Gamma_{R(L)}$, the current-phase relation takes the familiar Josephson form $J_{a}(\varphi)=J_{c} \sin \varphi$, where the critical current $J_{c}$ is determined by the smallest partial level width, $J_{c}=(e / \hbar) \Gamma_{R(L)}$ (the nonresonant critical current is proportional to $\Gamma_{0}^{2}$ ). Electron-vibron interactions strongly modify the resonant supercurrent. One readily finds three solutions of Eq. (6): $x_{c}=0$ and

$$
x_{c}(\varphi)= \pm \sqrt{\left(\frac{\lambda}{\hbar \omega_{0}}\right)^{2}-\left(\frac{\Gamma}{2 \lambda}\right)^{2}\left(\cos ^{2} \frac{\varphi}{2}+\gamma^{2} \sin ^{2} \frac{\varphi}{2}\right)},
$$

where $\Gamma=\Gamma_{L}+\Gamma_{R}, \gamma=\left(\Gamma_{L}-\Gamma_{R}\right) / \Gamma$. Note that the quasiclassical approximation holds for $x_{c} \gg 1$, i.e., for QD shifts much larger than the amplitude $x_{0}$ of zero-point fluctuations. One can see from Eq. (8) that the required inequality is always satisfied in the regime of strong interactions, $\lambda^{2} \gg \hbar \omega_{0} \Gamma_{0}$. In this case (or more precisely if $\lambda^{2}>\hbar \omega_{0} \Gamma_{0}$ for a symmetric junction) the energy minimum corresponds to $x_{c} \neq 0$, given by Eq. (8), and $x_{c}=0$ is an unstable solution (see the two-well potential in Fig. 1(b). The supercurrent (5) takes the form

$$
J_{\lambda}(\varphi)=e \omega_{0} \frac{\Gamma_{L} \Gamma_{R}}{2 \lambda^{2}} \sin \varphi
$$

which looks like a nonresonant Josephson current $\left(J_{c} \propto \Gamma_{0}^{2}\right)$. The conclusion that interactions always suppress the resonant critical current agrees with the results of Refs. 15 and 16. Notice that for strong interactions $\left(\lambda^{2} \gg \hbar \omega_{0} \Gamma_{0}\right)$ the suppression of the critical current (we will call it a "polaronic suppression") is power-law like, $J_{c} \propto \lambda^{-2}$, in distinction to the exponential suppression (Franck-Condon blockade) caused by the zero-point fluctuations of the QD coordinate in the regime of "hard" vibrons $\left(\hbar \omega_{0} \gg \Delta_{0}\right)$. Equation (9) can be physically interpreted as the standard formula for the nonresonant critical current (see, e.g., [23,13]) $J_{c} \simeq\left(e \Gamma_{0} / \hbar\right)\left(\Gamma_{0} /\left|\varepsilon_{0}\right|\right)$, where the level energy $\varepsilon_{0}$ is replaced by the polaronic energy $\varepsilon_{p} \simeq \lambda^{2} / \hbar \omega_{0} \gg \varepsilon_{0}$. In Sec. 5 we show that an analogous expression for the critical current can be derived by a Green's function approach.

A suppression of the critical current will be difficult to detect directly in an experiment since this requires an ability to vary the electron-vibron interaction strength. Therefore we will now consider how the interaction affects the current-phase relation. The interesting regime is that of weak or intermediate-to-strong interactions, $\lambda^{2} \lesssim \hbar \omega_{0} \Gamma_{0}$, when a variation of the phase difference $\varphi$ induces reentrant transitions of the vibrational subsystem from the "unshifted" $\left(x_{c}=0\right)$ to the "shifted" $\left(x_{c} \neq 0\right)$ state. One can see from Eq. (8) that for a symmetric junction and $\lambda^{2}=c \Gamma_{0} \hbar \omega_{0}$ (where $c<1$ is a numerical constant) the "shifted phase"

$$
x_{c}(\varphi)= \pm \sqrt{\frac{1}{c} \frac{\Gamma_{0}}{\hbar \omega_{0}}\left[c^{2}-\cos ^{2}(\varphi / 2)\right]}
$$

exists only in a finite interval of phase difference $(|\cos (\varphi / 2)|<c)$. For other values of $\varphi$ the symmetric coordinate position $\left(x_{c}=0\right)$ is realized. It is easy to check that at the transition points $\left(\varphi_{t}\right)$ the supercurrents calculated in the quasiclassical approximation for both phases coincide. However, in the vicinity of the transition points $\left(x_{c} \ll 1\right)$ we have to take into account quantum effects to correctly describe this region. In Fig. 2 we have plotted the $J-\varphi$ relation for a symmetric junction and medium-strong electron-vibron interaction $\left(\hbar \omega_{0} \Gamma_{0} / \lambda^{2}=1.1\right)$ by taking into account the zero-point energy of the vibrons in the new "shifted" vacuum given by Eq. (8) (polaritons in the terminology of Ref. 16). The vibron energy is then given by the expression

$$
\hbar \omega_{v}\left(x_{c}\right)=\hbar \omega_{0}-\frac{\lambda^{2} \Gamma_{0}^{2} \cos ^{2} \frac{\varphi}{2}}{E_{A}^{3}\left(x_{c}\right)} .
$$

Although the "unshifted phase" is not controlled by the quasiclassical approximation when $\varphi$ is close to $\varphi=\pi$, the reentrant transitions take place at the points $\varphi_{t}$ and $2 \pi-\varphi_{t}$ far away from the dangerous region. We see in Fig. 2 the appearance of special features (cusps) in the $J-\varphi$ curve at the transition points (see also Ref. 15).

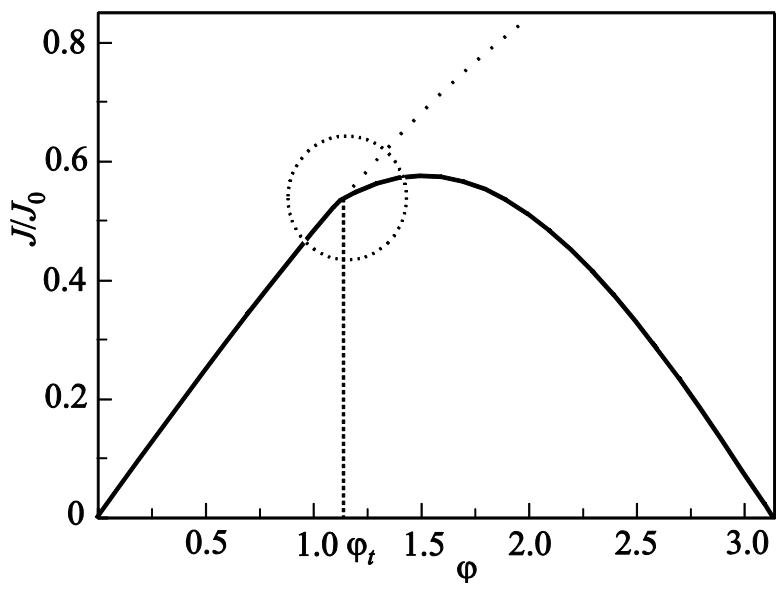

Fig. 2. Josephson current (normalized to $J_{0}=e \Gamma_{0} / \hbar$ ) plotted as a function of the superconducting phase difference $\varphi$ for $\hbar \omega_{0} \Gamma_{0} / \lambda^{2}=1.1$. The marked region indicates a cusp at $\varphi \approx 1.13$ in the current-phase curve. 
Another manifestation of vibrational effects is the hysteresis-like behavior of the supercurrent caused by phaseinduced transitions from the double- to the single-well potential. When the level energy $\left|\varepsilon_{0}\right| \gg \Gamma_{0}$ the effective double-well potential $E_{0}\left(x_{c}\right)$ becomes nonsymmetric. By using perturbation theory with respect to $\Gamma_{0}$ it is easy to obtain the following analytical expressions for the QD displacement and the critical current (for $\varepsilon_{0}<0$ )

$$
\begin{gathered}
x^{(l / g)} \simeq \pm \frac{\lambda}{\hbar \omega_{0}}\left\{1-\frac{1}{2}\left(\frac{\hbar \omega_{0} \Gamma_{0} \cos (\varphi / 2)}{\lambda^{2} \mp \hbar \omega_{0}\left|\varepsilon_{0}\right|}\right)^{2}\right\}, \\
J_{c}^{(l / g)} \simeq \frac{e \omega_{0}}{2} \frac{\Gamma_{0}^{2}}{\lambda^{2} \mp \hbar \omega_{0}\left|\varepsilon_{0}\right|},
\end{gathered}
$$

Here $(l / g)$ denote local and global minima. It is interesting to note that vibrational effects could enhance the critical current (13) compared to the nonresonant critical current $J_{c} \simeq\left(e \Gamma_{0} / \hbar\right)\left(\Gamma_{0} /\left|\varepsilon_{0}\right|\right)$ in the absence of electron-vibron interactions.

The global $(g)$ and local $(l)$ minima can be interchanged by a gate voltage pulse $V_{g}(t)$. The critical current corresponding to the local minimum is always larger than the one corresponding to the global minimum, $J_{c}^{(l)}>J_{c}^{(g)}$. For a special choice of model parameters (see Fig. 3) the barrier between the local and global minima disappears at $\varphi_{t}$ and $2 \pi-\varphi_{t}$ (see also [15]). If we neglect macroscopic quantum tunneling of the QD (the corresponding time scale is exponentially large in the limit $\omega_{0} \rightarrow 0$ since $\tau_{t} \propto \omega_{0}^{-1} \exp \left[U(\varphi) / \hbar \omega_{0}\right]$, where $U(\varphi)$ is the barrier height which weakly depends on $\omega_{0}$ ) the transitions from local to global minimum will occur only at transition points when the barrier disappears. Then one can imagine a hysteretic behavior of the Josephson current (see Fig. 3) caused by a gate voltage-induced transition in the vicinity of $\varphi=\pi$ and phase induced transitions at $\varphi_{t}$ and $2 \pi-\varphi_{t}$.

\section{Anomalous temperature behavior of critical current}

In the previous Section we showed that electron-vibron interactions tend to diminish the maximum supercurrent, which occurs on resonance, i.e., when $\varepsilon_{0}=0$. For strong interactions the critical current is greatly suppressed. A superconducting junction with a movable quantum-dot weak link in this case behaves as a strongly asymmetric Josephson junction and all interaction effects at zero temperature are hidden in a renormalization of the critical current. This polaronic suppression is lifted at high temperatures where it is natural to expect the standard $1 / T$ temperature scaling (see, e.g., Ref. 25) of the critical current to be recovered. In this Section we will show that in the presence of electron-vibron interactions the cross-over from the low- $T$ to the high- $T$ regime is nonmonotonic

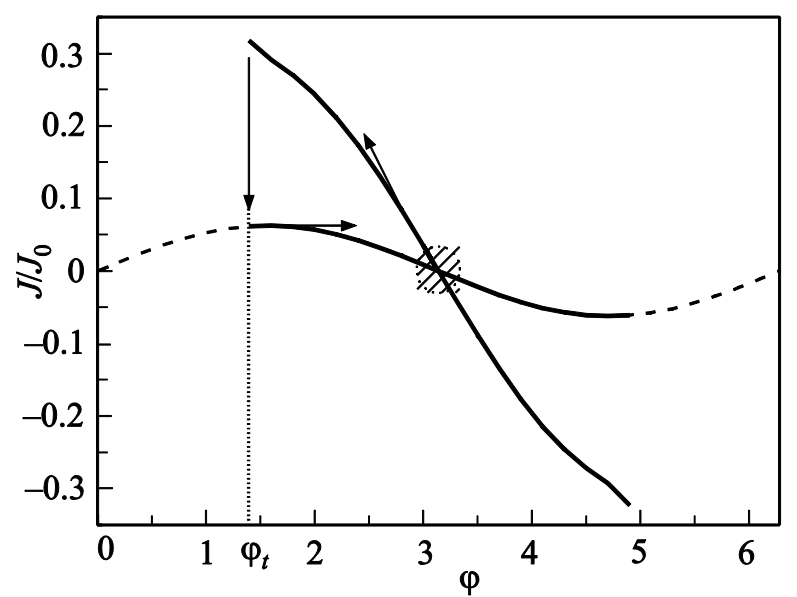

Fig. 3. Hysteretic behavior of the Josephson current plotted as a function of the superconducting phase difference $\varphi$ for $\hbar \omega_{0} \Gamma_{0} / \lambda^{2}=0.2$ and $\varepsilon_{0} \hbar \omega_{0} / \lambda^{2}=0.6$. In the vicinity of $\varphi=\pi$ the quantum dot is transferred to the local potential energy minimum (see text) by a gate-voltage pulse. The system remains in the local minimum up to $\varphi=\varphi_{t}$ (or $\varphi=2 \pi-\varphi_{t}$ ), where the barrier between the two minima disappears and the system "falls" to the global potential energy minimum.

with a maximum critical current at temperatures of the order of the polaron energy, $T_{p} \simeq \lambda^{2} / \hbar \omega_{0}$.

At finite temperatures the Josephson current is determined by the $\varphi$-derivative of the grand canonical potential $\Omega$, which in the quasiclassical approach comprises three terms: the elastic (potential) energy of the vibrational subsystem, the grand canonical potential of the vibron excitations and the grand canonical potential of the two-level system formed by Andreev bound states. Hence

$$
\begin{gathered}
\Omega=\frac{\hbar \omega_{0}}{2} x_{c}^{2}+T \ln \left\{1-\exp \left(-\frac{\hbar \omega_{v}\left(x_{c}\right)}{T}\right)\right\}- \\
-2 T \ln \left[2 \cosh \left(\frac{E_{A}\left(x_{c}\right)}{2 T}\right)\right],
\end{gathered}
$$

where $E_{A}$ is determined by Eq. (4) (for simplicity we consider symmetric junction $\left.\Gamma_{L}=\Gamma_{R}=\Gamma_{0}\right)$ and $\hbar \omega_{v}\left(x_{c}\right)$, given by Eq. (11), is the vibron energy in the state with a shifted coordinate $x_{c}$. The equilibrium coordinate $x_{c}=x(\varphi, T)$ is found from the extremum equation $\partial \Omega / \partial x_{c}=0$. Using this equation it is easy to express the Josephson current in the simple form

$$
J(T)=\frac{2 e}{\hbar} \frac{\partial \Omega}{\partial \varphi}=\frac{e}{2 \hbar} \frac{\Gamma_{0}^{2}}{E_{A}\left(x_{c}\right)} \tanh \left[\frac{E_{A}\left(x_{c}\right)}{2 T}\right] \sin \varphi
$$

The equilibrium QD coordinate is plotted as a function of temperature for different electron-vibron coupling strengths in Fig. 4. The upper part of each curve corresponds to the potential minimum and the lower part corresponds to the local maximum of $\Omega$ (these states are abso- 


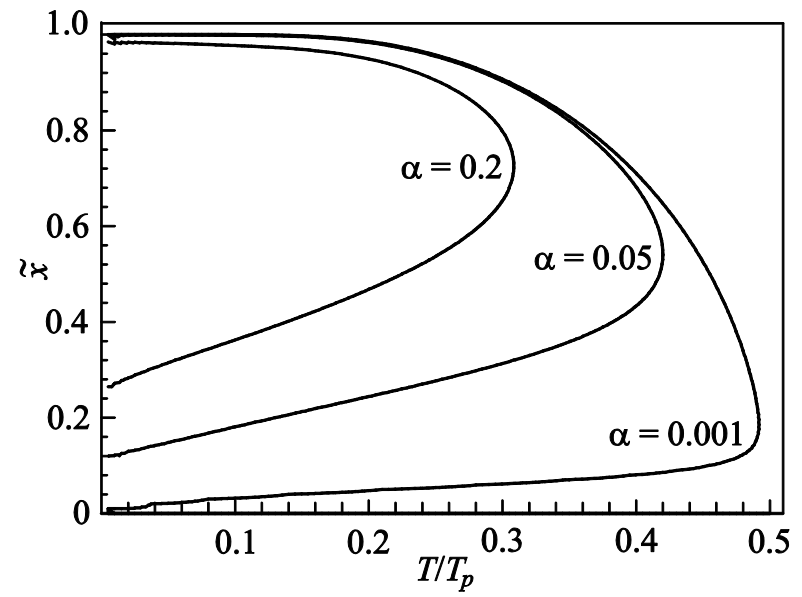

Fig. 4. Dimensionless equilibrium coordinate for the weak-link quantum-dot, $\tilde{x}=x_{c} \hbar \omega_{0} / \lambda$, plotted as a function of dimensionless temperature, $T / T_{p}=T \hbar \omega_{0} / \lambda^{2}$, for $\varphi=1, \quad \hbar \omega_{0} / \lambda=0.25$, and different values of $\alpha=\hbar \omega_{0} \Gamma_{0} / \lambda^{2}$.

lutely unstable). We see that an increasing temperature tends to decrease the average coordinate and for temperatures $T>T_{m}$ the only solution of the extremum equation is $x_{c}=0$. The "critical" temperature $T_{m}$ and the corresponding "critical" coordinate $x_{m}$ are found from the two equations $\partial \Omega\left(x_{m}, T_{m}\right) / \partial x_{c}=0$ and $\partial x_{c}\left(x_{m}, T_{m}\right) / \partial T=\infty$, which for temperatures $T \gg \hbar \omega_{0}$ takes the form

$$
\left\{\begin{array}{c}
\tilde{x}-\tanh \left(\frac{\tilde{x}}{2 T}\right)+3 \alpha_{\varphi}^{2} \frac{T}{\tilde{x}^{4}}=0, \\
1-\frac{1}{2 T} \cosh ^{-2}\left(\frac{\tilde{x}}{2 T}\right)-12 \alpha_{\varphi}^{2} \frac{T}{\tilde{x}^{5}}=0 .
\end{array}\right.
$$

Here $\quad \alpha_{\varphi} \equiv \hbar \omega_{0} \Gamma_{\varphi} / \lambda^{2}<1, \quad \Gamma_{\varphi}=\Gamma_{0}|\cos (\varphi / 2)| \quad$ and $\tilde{x}=x_{m} \hbar \omega_{0} / \lambda, \quad T=T_{m} \hbar \omega_{0} / \lambda^{2}$. It is straightforward to show that in the strong interaction limit $\left(\alpha_{\varphi} \rightarrow 0\right)$ the asymptotic solution of Eq. (16) is $x_{m} \simeq C_{1} \alpha_{\varphi}^{2 / 7}$, $T_{m} \simeq\left(\lambda^{2} / 2 \hbar \omega_{0}\right)\left(1-C_{2} \alpha_{\varphi}^{4 / 7}\right)$, where $C_{1,2}$ are numerical constants of order 1. From Fig. 4 it is clear that for intermediate-to-strong interactions $\left(1<\lambda^{2} / \hbar \omega_{0} \Gamma_{0} \lesssim 10^{2}\right)$ the transition temperature $T_{m}$ is of the order of the polaronic energy $T_{m} \simeq \lambda^{2} / \hbar \omega_{0}$ and the corresponding coordinate $x_{m} \simeq \lambda / \hbar \omega_{0} \gg 1$. The transition to the regime of resonant tunneling $\left(x_{c}=0\right)$ in this case occurs abruptly $(\Delta x \gg 1)$ and is manifest in a jump-like dependence of the critical current on temperature (see Fig. 5).

With an increase of temperature the QD is shifted towards its symmetrical coordinate position in the junction and hence up to temperatures $T \leq T_{m}$ the Josephson current is monotonically increased. Note that the temperature enhancement of the current is entirely due to the vibronic excitations (second term in Eq. 14). Without this contribution to $\Omega$ the Josephson current would be temperature independent. For temperatures $T \ll \lambda^{2} / \hbar \omega_{0}$ one can de-

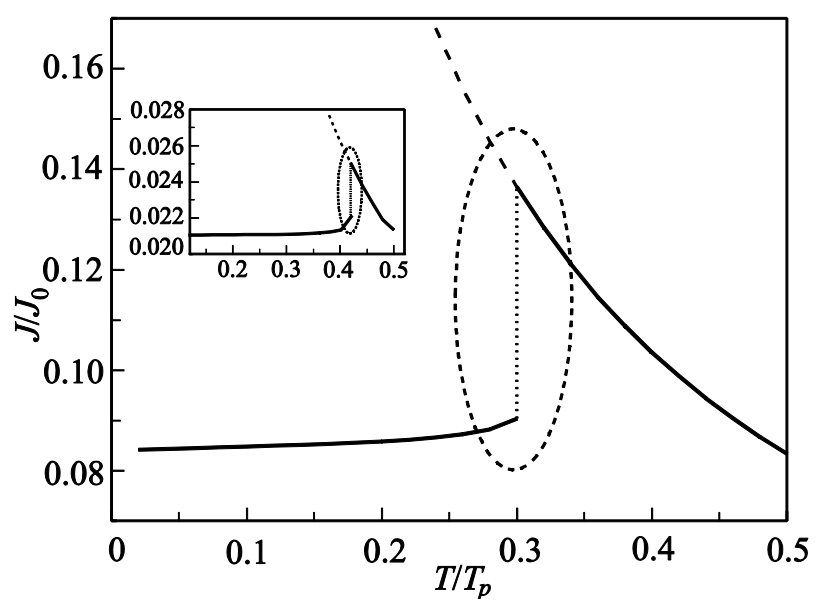

Fig. 5. Temperature dependence of the Josephson current for $\varphi=1$, $\hbar \omega_{0} / \lambda=0.25, \varepsilon_{0}=0$, and $\alpha=0.2$ (inset: $\alpha=0.05$ ). The current is normalized to the maximum critical current $J_{0}=e \Gamma_{0} / \hbar$, and temperature to the polaron energy $T_{p}=\lambda^{2} / \hbar \omega_{0}$.

rive an analytical expression for the temperature dependence of the Josephson current,

$$
J(T) \simeq J_{\lambda}\left[1+3 \cos ^{2} \frac{\varphi}{2}\left(\frac{\Gamma_{0} \omega_{0}}{\lambda^{2}}\right)^{2}\left(\frac{\omega_{0}}{\lambda}\right)^{2} n_{B}\left(\omega_{0}\right)\right] \sin \varphi,
$$

$n_{B}\left(\omega_{0}\right)=1 /\left[\exp \left(\hbar \omega_{0} / T\right)-1\right], \quad J_{\lambda}=\left(e \Gamma_{0} / 2 \hbar\right)\left(\hbar \omega_{0} \Gamma_{0} / \lambda^{2}\right)$. Temperature corrections are exponentially small as long as $T \ll \hbar \omega_{0}$ while the current increases linearly with temperature in the interval $\hbar \omega_{0} \ll T \ll \lambda^{2} / \hbar \omega_{0}$. At high temperatures, $T>T_{m}$, the junction is in the state $x_{c}=0$ (i.e., in the regime of resonant tunneling if $\varepsilon_{0}=0$ ) and the Josephson current is

$$
J_{r}(T)=\frac{e \Gamma_{0}}{\hbar} \sin \left(\frac{\varphi}{2}\right) \operatorname{sgn}\left[\cos \left(\frac{\varphi}{2}\right)\right] \tanh \left(\frac{\Gamma_{0}|\cos (\varphi / 2)|}{2 T}\right) .
$$

In the general case of a finite $\left|\varepsilon_{0}\right| \ll \varepsilon_{p}$ the supercurrent in the high temperature region reads (see, e.g., $[23,24]$ )

$$
J(T)=\frac{e \Gamma_{0}^{2}}{2 \hbar} \frac{\sin \varphi}{\sqrt{\varepsilon_{0}^{2}+\Gamma_{\varphi}^{2}}} \tanh \left(\frac{\sqrt{\varepsilon_{0}^{2}+\Gamma_{\varphi}^{2}}}{2 T}\right) .
$$

In the quasiclassical approximation the currents in the low$T$ and high- $T$ "phases" are not matched at $T=T_{m}$ and here one expects a "jump" of the critical current (see Fig. 5, where $\varepsilon_{0}=0$ and the expected effect is maximal). In our approach the jump is of the order of the critical resonant current.

Could quantum corrections and thermal fluctuations smear out the nonanalytical behavior in the transition region? In the state where $x_{c} \gg 1$ the quantum fluctuations 
are small and can be neglected. On the contrary, quantum fluctuations could certainly modify the resonant critical current $\left(x_{c}=0\right)$. This question will be considered in Sec. 5 by using a Green's function approach. We will show that in the regime of strong coupling, $\lambda^{2} \gg \hbar \omega_{0} \Gamma_{0}$, the critical current does demonstrate an anomalous temperature behavior with a maximum at $T_{m} \sim \varepsilon_{p}$. The effect is, however, quantitatively small. Nevertheless the temperature dependence of the critical current significantly differs from the standard tanh $\left(\varepsilon_{0} / 2 T\right)$-dependence (see Fig. 8).

Although the quasiclassical approximation can not quantitatively describe the transition region $\left(T \sim \varepsilon_{p}\right)$ it has certain advantages compared to the quantum field approach. The calculations in a simple model Eqs. (1)-(3) can easily be generalized to the more realistic case when the electron tunneling amplitudes are coordinate-dependent quantities and the vibrations of QD are strongly anharmonic.

\section{Coordinate-dependent tunneling amplitudes}

In the previous Section we studied the influence of polaronic effects on the Josephson current in a model where the electron tunneling matrix elements were assumed to be coordinate-independent. Large shifts of the QD inside the junction could, however, strongly affect tunneling probabilities. This would be the case, e.g., if a movable quantum dot (molecule) is placed in the gap between two superconducting electrodes as in Fig. 1(b). In this situation it is reasonable to model the coordinate dependence of the tunneling amplitudes as (see, e.g., [6])

$$
t_{j}(\hat{x})=t_{0 j} \exp \left(j \lambda_{t} \hat{x}\right)
$$

where $j=(L, R)=(+,-)$ and $\lambda_{t}=x_{0} / l_{t} \quad\left(l_{t}\right.$ is the electron tunneling length). In addition, for large thermal fluctuations shifts one has to take into account the effects of anharmonicity of the vibration potential, which now is modeled by the expression

$$
V_{b}(x)=\frac{\hbar \omega_{0}}{a^{2}}[\cosh (a x)-1] .
$$

Here $\omega_{0}$ is the vibrons frequency (for $a \rightarrow 0$ ) and $a=x_{0} / W, W$ is a length that characterizes the steepness of the confining potential. A more realistic model would be a sum of two Morse potentials separated by some distance $d_{0}$. Such a potential is double-well-like and for small QD displacements it describes a strongly asymmetric Josephson junction. Here we consider the situation when $\Gamma_{L} \simeq \Gamma_{R}$ and therefore we will use the single-well potential (21). It will be shown that qualitatively the temperature behavior of the critical current is not sensitive to the concrete form of the confining potential.

In the quasiclassical approach the ground state energy of the weak link now takes the form

$$
\begin{gathered}
E_{0}\left(x_{c}, \varphi\right)=\frac{\hbar \omega_{0}}{a^{2}}\left[\cosh \left(a x_{c}\right)-1\right]- \\
-\sqrt{\left(\varepsilon_{0}+\lambda x_{c}\right)+\frac{1}{4}\left(\Gamma_{L}^{2} \mathrm{e}^{-2 \lambda x_{c}}+\Gamma_{R}^{2} \mathrm{e}^{2 \lambda x_{c} x_{c}}+2 \Gamma_{L} \Gamma_{R} \cos \varphi\right)} .
\end{gathered}
$$

This energy is bounded from below only when $a>\lambda_{t}$, otherwise the energy minima correspond to $x_{c} \rightarrow \pm \infty$. Physically this would mean that the QD is "glued" to one of the electrodes and that the mechanical motion is frozen.

The displacement of the QD can be found from the equation $\partial E_{0} / \partial x_{c}=0$. In the region $a \gtrsim \lambda_{t} \gtrsim 1$, where the tunneling rates are strongly renormalized by the displacement of QD, the equilibrium coordinate position $x_{c}$ in the considered limit $\Gamma_{L, R} \gg \hbar \omega_{0}$ is

$$
x_{c} \simeq \frac{1}{a-\lambda_{t}} \ln \left(a \lambda_{t} \frac{\Gamma_{R(L)}}{\hbar \omega_{0}}\right) \gg 1, \quad \Gamma_{R(L)}>\Gamma_{L(R)} .
$$

We see that in the limit $\omega_{0} \rightarrow 0$ the dot displacement depends neither on the superconducting phase $\varphi$ nor on the interaction constant $\lambda$. The QD is shifted towards the nearest electrode in order to enhance the contribution of the negative energy Andreev level (and maximize the cohesive energy) (see also [26]). The junction behaves as a strongly asymmetric tunnel junction supporting a nonresonant supercurrent $J\left(\varphi, \omega_{0}\right)=J_{c}\left(\omega_{0}\right) \sin \varphi$, where

$$
J_{c}\left(\omega_{0}\right) \simeq \frac{e \Gamma_{L(R)}}{\hbar}\left(\frac{1}{a \lambda_{t}} \frac{\hbar \omega_{0}}{\Gamma_{R(L)}}\right)^{\frac{\lambda_{t}}{a-\lambda_{t}}}, \quad \Gamma_{R(L)}>\Gamma_{L(R)} .
$$

Next we will discuss the temperature dependence of this critical current, noting that we again expect it to be nonmonotonic.

At finite temperatures there are two oppositely directed tendencies, which influence the supercurrent. The population of the positive energy Andreev level $\left(+E_{A}\right)$ at $T \neq 0$ tends to decrease the Josephson current and at temperatures much larger then the Andreev level spacing the Josephson current scales as $1 / T$. On the other hand thermally excited vibrons at $T \gg \hbar \omega_{0}$ diminish the average coordinate $x_{c}(T)<x_{c}(0)$ and thus at low temperatures the supercurrent is enhanced. Therefore we expect an anomalous temperature dependence of critical current analogous to the results of Sec. 3. Numerical calculations support our physical arguments and (see Fig. 6) show that qualitatively the temperature dependence of the Josephson current in the adiabatic regime $\omega_{0} \rightarrow 0$ is not sensitive to the shape of the confining potential and the choice of bare tunneling amplitudes. In our quasiclassical approach the supercurrent experiences a jump at some critical temperature which is 


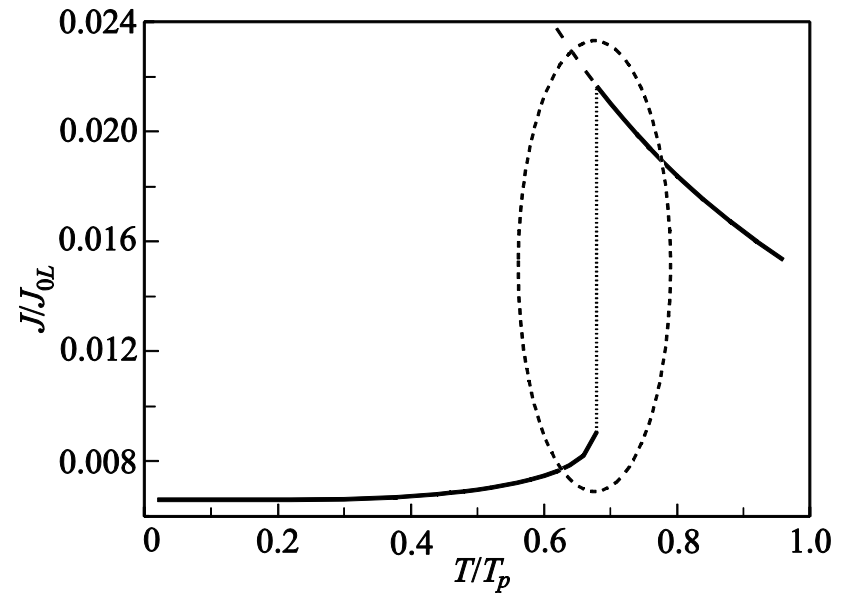

Fig. 6. Temperature dependence of the Josephson current in a model system with coordinate-dependent tunneling matrix elements plotted for $\varphi=1, \quad \hbar \omega_{0} / \lambda=0.05, \quad \hbar \omega_{0} \Gamma_{R} / \lambda^{2}=0.07$, $\hbar \omega_{0} \Gamma_{L} / \lambda^{2}=0.04, \quad a \lambda / \hbar \omega_{0}=11$, and $\lambda_{t} \lambda / \hbar \omega_{0}=8$. The current is normalized to $\left.J_{0 L}=e \Gamma_{L} / \hbar\right)$ and temperature to $T_{p}=\lambda^{2} / \hbar \omega_{0}$.

the result of a transition of the QD from a strongly asymmetric $\left(x_{c} \gg 1\right)$ to a symmetric position $\left(x_{c}=0\right)$.

\section{Temperature enhanced Josephson current. Green's function approach}

In this Section we will calculate the temperature dependence of the critical current by using a Green's function approach. We are interested in the strong coupling regime of the model defined by Eqs. (1)-(3). In this case it is useful (see, e.g., [27]) to perform a unitary transformation using

$$
U=\exp \left(-g\left(b^{\dagger}-b\right) \sum_{\sigma=\uparrow, \downarrow} d_{\sigma}^{\dagger} d_{\sigma} / \sqrt{2}\right)
$$

where $g=-\lambda / \hbar \omega_{0}$ is the dimensionless electron-vibron interaction constant. This transformation eliminates the electron-vibron interaction from the Hamiltonian (1) and shifts the level energy $\varepsilon_{0} \rightarrow \varepsilon_{p s}=\varepsilon_{0}-g^{2} \hbar \omega_{0} / 2$ (polaronic shift) while changing the electron-electron correlation energy so that $U \rightarrow U_{\text {eff }}=U-g^{2} \hbar \omega_{0}$ [13]. The electron-vibron interaction term reappears in the tunneling Hamiltonian (3), where the tunneling amplitude $t_{0 j}$ is replaced by the operator $t_{0 j}(\hat{p})=t_{0 j} \exp (i g \hat{p}) \quad\left(\hat{p}=i\left(b^{\dagger}-b\right) / \sqrt{2}\right)$.

The nonequilibrium Green's function method (Keldysh technique) allows one to express the electrical current through an interacting quantum dot in terms of retarded Green's functions of the dot levels. For normal metal (noninteracting) electrodes this formula is known as MeirWingreen formula [19]. Its generalization to superconducting leads was reported in Ref. 18. Here we consider a simplified form of the Josephson current [Eq. (30) in Ref. 18] when the superconducting order parameter is considered to be the largest energy in the problem (formally $\Delta_{0} \rightarrow \infty$ ). The current in this limit reads

$J=-\frac{2 e \Gamma_{0}^{2}}{\hbar} \int d \varepsilon f(\varepsilon) \operatorname{Im}\left\{\frac{1}{\left(g_{11}^{(r)-1} g_{22}^{(r)-1}\right)^{*}-\Gamma_{\varphi}^{2}}\right\} \sin \varphi$

where $f(\varepsilon)$ is the Fermi distribution function, $\varphi$ is the superconducting phase difference, and $g_{\alpha \beta}^{(r)}$ are the retarded Green's function of the QD in the Nambu representation and $\Gamma_{\varphi}=\Gamma_{0} \cos (\varphi / 2)$. For a QD without interactions $(g=0$, $\left.U_{\text {eff }}=0\right) . \quad g_{11(22)}^{r}=\left(\varepsilon \mp \varepsilon_{0}+i 0\right)^{-1}$ one recovers the standard expression (19) for the Josephson current through a resonant level $\left(\left|\varepsilon_{0}\right| \ll \Delta_{0}\right)$ from Eq. (25).

To proceed further we neglect the effective electronelectron interaction and let $U_{\text {eff }} \simeq 0$ in the transformed QD Hamiltonian. Note that the contribution of the electronvibron interaction to the electron-electron correlations always suppresses the effects of Coulomb repulsion, which depends on the the effective capacitance $C_{\mathrm{eff}}$ of the QD. Therefore it is possible to minimize $U_{\text {eff }}$ by changing $C_{\text {eff }}$.

Even without electron-electron correlations the Green's function $g_{\alpha \beta}^{r}(\varepsilon)$ in our model can not be evaluated exactly. One needs additional approximations to calculate the Josephson current from Eq. (25). We will use the "polaron tunneling approximation" [20] where the electron propagators are replaced by polaron Green's functions,

$$
\begin{gathered}
\left\langle d_{\sigma}^{(p)}(t) d_{\sigma}^{(p) \dagger}\right\rangle \equiv\left\langle d_{\sigma}(t) d_{\sigma}^{\dagger} \exp [-i g \hat{p}(t)] \exp [i g \hat{p}]\right\rangle \approx \\
\approx\left\langle d_{\sigma}(t) d_{\sigma}^{\dagger}\right\rangle_{f}\left\langle\mathrm{e}^{[-i g \hat{p}(t)]} \mathrm{e}^{[i g \hat{p}]}\right\rangle_{b} .
\end{gathered}
$$

Here the symbol $\langle\ldots\rangle_{f, b}$ stands for a thermodynamic average with respect to noninteracting fermionic (f) and bosonic (b) Hamiltonians. Factorization of boson and fermion averages is always justified in perturbation theory to lowest order in the level width $\Gamma_{0}$. Beyond perturbation theory the "polaron tunneling approximation" [20] holds when the characteristic time of polaron formation $\left(\hbar / \varepsilon_{p} \sim 1 / g^{2} \omega_{0}\right)$ is much shorter than the electron "lifetime" in the QD $\left(\sim \hbar / \Gamma_{0}\right)$, i.e., in the regime of strong coupling $g^{2} \gg \Gamma_{0} / \hbar \omega_{0}$. This criterion coincides with the one considered in Sec. 2, where the quasiclassical approximation was studied.

In the polaron tunneling approximation the desired retarded Green's functions in the Nambu representation,

$$
g_{\alpha \beta}^{r}=-i \theta(t)\left(\begin{array}{cc}
\left\langle\left\{d_{\uparrow}^{(p)}(t), d_{\uparrow}^{(p) \dagger}\right\}\right\rangle & 0 \\
0 & \left\langle\left\{d_{\downarrow}^{(p) \dagger}(t), d_{\downarrow}^{(p)}\right\}\right\rangle
\end{array}\right),
$$

take the form (after switching to the energy representation by taking a Fourier transform) 


$$
\begin{gathered}
g_{11(22)}^{r}=\exp \left[-g^{2}\left(1+2 n_{B}\right)\right] \times \\
\times \sum_{n=-\infty}^{\infty} \frac{\exp \left(-\frac{n \hbar \omega_{0}}{2 T}\right) I_{n}\left[2 g^{2} \sqrt{n_{B}\left(1+n_{B}\right)}\right]}{\varepsilon \mp \varepsilon_{p s} \pm n \hbar \omega_{0}+i 0}
\end{gathered}
$$

where $n_{B}=1 /\left[\exp \left(\hbar \omega_{0} / T\right)-1\right]$ is the Bose-Einstein distribution function and $I_{n}(z)$ is the modified Bessel function of the first kind.

At low temperatures, $T \ll \hbar \omega_{0}$, the argument of the Bessel functions in Eq. (28) is small, $I_{n}(z \rightarrow 0) \approx$ $\approx(z / 2)^{|n|} / \Gamma(n+1)$ [28], and Eq. (28) simplifies to read

$$
g_{11(22)}^{r}(\varepsilon ; T=0)=\exp \left(-g^{2}\right) \sum_{n=0}^{\infty} \frac{g^{2 n}}{n !\left(\varepsilon \mp \varepsilon_{p s} \mp n \hbar \omega_{0}+i 0\right)} .
$$

It is easy to see that the corresponding spectral function, $A(\varepsilon)=-2 \operatorname{Im}\left[g^{r}(\varepsilon)\right]$, is a series of $\delta$-function peaks (spaced by $\hbar \omega_{0}$ ) with a Poisson distribution of weights. Note that for a strong interaction, $g \gg 1$, many terms (up to $n$ of the order of $g^{2}$ ) contribute to the sum in Eq. (29).

When evaluating the normal current (or conductance) by using the Meir-Wingreen formula the difference of the Fermi distribution functions "cuts out" a finite interval $(\sim e V)$ in the integral over energies $\varepsilon$. When $e V<\hbar \omega_{0}$ only the the "elastic peak" $(n=0)$ survives after integration. The weight of the elastic peak is exponentially suppressed, which is how the Franck-Condon blockade is manifested

When evaluating the supercurrent from Eq. (25) we have to sum over all $n>0$ in the considered limit $\Delta_{0} \gg \hbar \omega_{0}$. Actually the required inequality for $g \gg 1$ is reduced to $g^{2} \hbar \omega_{0} \lesssim \Delta_{0}$ since for $n>n_{m}=\left[g^{2}\right]$ the series in Eq. (29) quickly converges. Then we can approximate the Green's function by the simple expression

$$
g_{11(22)}^{r}(\varepsilon) \approx \frac{1}{\left(\varepsilon \mp \varepsilon_{p s} \mp g^{2} \hbar \omega_{0}+i 0\right)},
$$

which after substitution into Eq. (25) results in a Josephson current of the form (19) at $T=0$ but with $\varepsilon_{0}$ replaced by $\varepsilon_{p s}+g^{2} \hbar \omega_{0}=\varepsilon_{0}+g^{2} \hbar \omega_{0} / 2$. Note that in the region of model parameters where the polaron tunneling approximation is valid $\left(g^{2} \hbar \omega_{0} \gg \Gamma_{0}\right)$ we can neglect the term $\Gamma_{\varphi}=\Gamma_{0} \cos (\varphi / 2)$ in Eqs. (25) and (19) and the critical current coincides with the corresponding expression, Eq. (9), derived in the quasiclassical approximation. The unrestricted summation over $n$ in Eq. (29) means that the exponential Franck-Condon blockade is lifted by virtual emission and absorbtion of vibrons. The remaining polaronic (power-law) suppression of the Josephson current in a S-QD-S junction is analogous to the Franck-Condon blockade of co-tunneling in normal transport through a vibrating QD [2]. Note that although in our derivation of the vibrationally mediated Josephson current we cannot consider the limit of resonant tunneling $\left(\varepsilon_{p s} \lesssim \Gamma_{0}\right)$, the exponential suppression of the resonant Josephson current found in Ref. 14 is difficult to verify for the case of soft vibrons $\hbar \omega_{0} \ll \Delta_{0}$, when virtual vibron processes strongly modify the QD Green's function.

Now we turn to studying the temperature dependence of the nonresonant critical current [see Eqs. (25) and (28)]

$$
\begin{gathered}
J_{c}(T)=\frac{e \Gamma_{0}^{2}}{\hbar} \exp \left[-2 g^{2} \operatorname{coth}(x)\right] \times \\
\times \sum_{n, l=-\infty}^{\infty} \frac{\mathrm{e}^{-(n+l) x} I_{n}\left(\frac{g^{2}}{\sinh (x)}\right) I_{l}\left(\frac{g^{2}}{\sinh (x)}\right)}{2 \varepsilon_{p s}-\hbar \omega_{0}(n+l)}\left(f_{l}^{-}-f_{n}^{+}\right),
\end{gathered}
$$

where $x=\hbar \omega_{0} / 2 T$ and $f_{j}^{ \pm}=f\left[ \pm\left(\varepsilon_{p s}-j \hbar \omega_{0}\right)\right]$. At low temperatures it is easy to recover Eq. (9) from Eq. (31). The high temperature region can be considered by using the well known exact formula for the summation of Bessel functions (see, e.g., [28])

$$
\sum_{k=-\infty}^{\infty} t^{k} I_{k}(z)=\mathrm{e}^{\frac{z}{2}\left(t+\frac{1}{t}\right)}
$$

With the help of this formula it is easy to evaluate the high temperature asymptotics of Eq. (31). It reads

$$
J_{c}\left(T \gg g^{2} \hbar \omega_{0}\right) \simeq \frac{e \Gamma_{0}^{2}}{4 \hbar T}\left(1-\frac{1}{3} \frac{g^{2} \hbar \omega_{0}}{T}\right) .
$$

We see that when the thermal energy is much larger than the polaronic energy the polaronic suppression of the Josephson

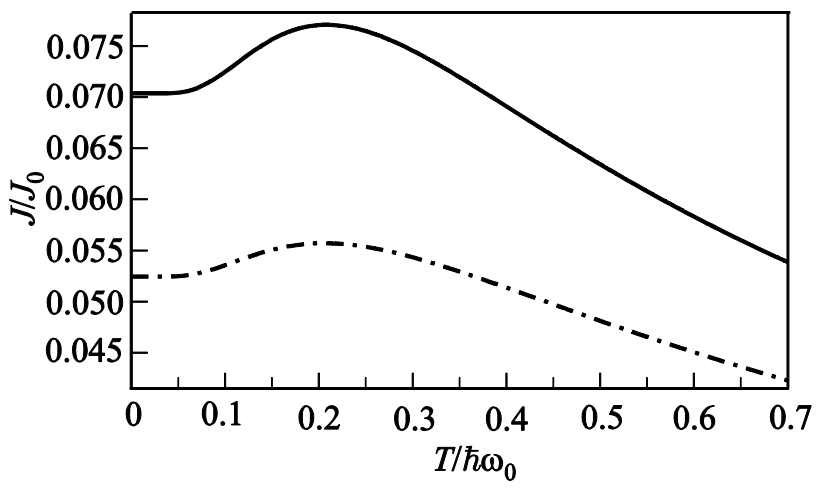

Fig. 7. Temperature dependence of the Josephson current normalized to $J_{0}=e \Gamma_{0} / \hbar$ and evaluated for $\Gamma_{0} / \hbar \omega_{0}=0.3$ and $\varepsilon_{0} / \hbar \omega_{0}=0.1$. The solid (dash-dotted) curve is the result for $g=1.7 \quad(g=2.2) . \quad J_{0}$ is the maximum zero-temperature value of the current, which obtains at resonance, i.e., for $\varepsilon_{0}=0$, without electron-vibron interactions $(g=0)$. 
current is lifted and the critical current scales as $1 / T$. Numerical calculations show (see Fig. 7) that in the region $T \lesssim \hbar \omega_{0}$ the temperature dependence of the critical current is anomalous - the Josephson current is enhanced by an increase of temperature. The supercurrent peaks at a temperature of the order of the vibron energy. Our calculations in the polaronic tunneling approximations did not reveal a particularly pronounced temperature enhancement of the supercurrent since the ratio $\left(J_{\max }-J_{\min }\right) / J_{\min } \lesssim 0.1$ is always small. However, this ratio tends to increase as one approaches (by varying $\varepsilon_{0}$ ) the region of resonant tunneling, which can not be correctly described in the polaron tunneling approximation.

In Fig. 8 we have plotted the temperature dependence of critical current normalized by the standard result, $\tanh (\varepsilon / 2 T)$. We see that the temperature dependence of the normalized current is anomalous. At low temperatures the lifting of the polaronic suppression results in a temperature-enhanced supercurrent. At high temperatures the Josephson current approaches its standard 1/T asymptotics from below (see Eq. (33)). This leads to a pronounced nonmonotonic behavior of the normalized supercurrent with a maximum and a minimum in a large temperature interval.

\section{Conclusions}

We have investigated vibrational effects on the Josephson current through a single-level $\left(\varepsilon_{0}\right)$ quantum-dot weak link between two bulk superconductors when the dot interacts with a vibrational mode $\left(\omega_{0}\right)$.

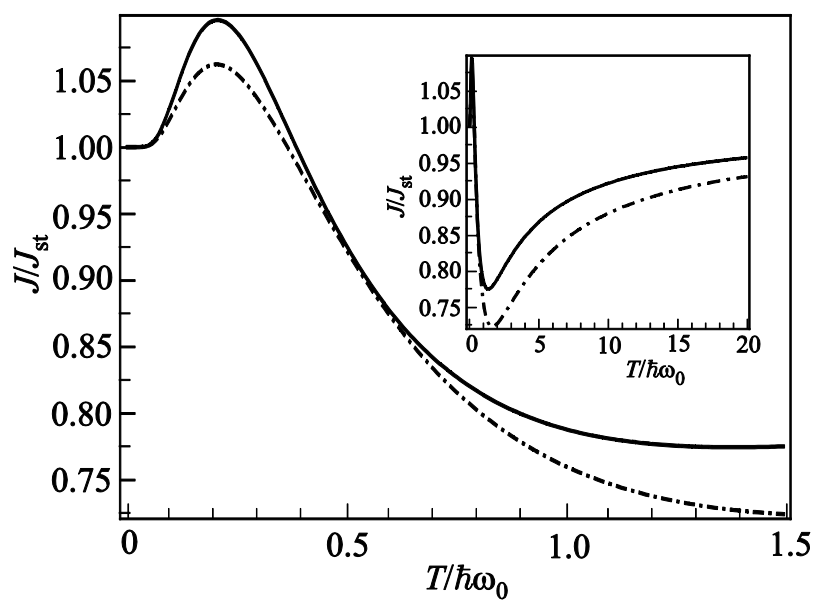

Fig. 8. Temperature dependence of the Josephson current normalized to the standard temperature-dependent expression $J_{\text {st }}=\left(e \Gamma_{0}^{2} / 2 \hbar \tilde{\varepsilon}\right) \tanh (\tilde{\varepsilon} / 2 T)$. Here $\tilde{\varepsilon}$ is determined by the critical current at zero temperature $\left(\tilde{\varepsilon}=e \Gamma_{0}^{2} / 2 \hbar J_{c}(T=0)\right)$ in order for the normalized current to be unity at $T=0$. At high temperatures the normalized Josephson current saturates to unity from below (see inset). Deviations from unity at low and intermediate temperatures indicate an anomalous temperature dependence of the Josephson current.
It is physically evident that an electron-vibron coupling should tend to suppress the critical Josephson current. Indeed, for different regimes of coherent electron transport it has been demonstrated $[13,15,16]$ that the Josephson current is influenced by the electron-vibron interaction at low temperatures, $T \ll \hbar \omega_{0}$. However, these results do not necessarily facilitate an experimental detection of vibrational effects on the Josephson current since these are "hidden" in a multiplicative renormalization of unknown bare tunneling probabilities.

In this work, on the other hand, we have shown that electron-vibron interactions give rise to polaronic effects manifested in an anomalous temperature dependence of the critical current, which is both measurable and significantly different from the standard $\tanh \left(\varepsilon_{0} / 2 T\right)$-behavior. We find that interactions with a low-energy vibrational mode $\left(\hbar \omega_{0} \ll \Delta\right)$ lead to a small anomalous increase of the critical current with increasing temperature at low temperatures $\left(T<T_{m}\right)$, a significant increase of the critical current at $T \sim T_{m}$ and for higher temperatures a decrease of the current, which becomes proportional to $1 / T$ (approaching the standard result from below) at high temperatures, $T \gg T_{m}$, when the polaronic suppression of the Josephson current is completely lifted. The characteristic temperature $T_{m}$ is of the order of the polaronic energy shift $\lambda^{2} / \hbar \omega_{0}=g^{2} \hbar \omega_{0}$, which depends on the strength of the electron-vibron interaction - either characterized by the interaction energy $\lambda$ or the dimensionless electron-vibron coupling constant $g$. This temperature behavior is analogous to what one finds in normal electron transport, where the influence of vibrational effects on the conductance leads to an anomalous temperature scaling of the peak conductance in the regime of sequential electron tunneling. It is interesting to note that the effect of a temperature enhancement is neither sensitive to the choice of vibron potential (which could be strongly anharmonic) nor to a possible coordinate dependence of the tunneling amplitudes.

We conclude that the predicted anomalous temperature dependence of the critical Josephson current is a distinct signature of polaronic effects on the Josephson current. It is easy to estimate the transition temperature $T_{m}$ by using known experimental parameters for normal electron tunneling through (i) a suspended single wall carbon nanotube (SWNT) (see, e.g., [29]) and (ii) a $\mathrm{C}_{60}$-based molecular transistor [30]. In experiments with a suspended SWNT it has been shown [29] that the electron-vibron interaction is strong with a dimensionless coupling constant $g$ of about 1-3. Using data from Ref. 29 one finds that the characteristic "polaronic" temperature is $T_{m} \simeq 1 \mathrm{~K}$ for a $1 \mu \mathrm{m}$ long SWNT coupling to the stretching mode or for a $100 \mathrm{~nm}$ long SWNT coupling to the bending mode. This means that the polaronic energy scale falls within the range of typical energies of weak links between ordinary low- $T$ superconductors. For a fullerene-based quantum dot the characteristic temperature is $T_{m} \sim 10 \mathrm{~K}$. 
In summary we have shown that in a suspended SWNTs the interplay of polaronic and superconducting effects results in an unusual temperature dependence of the critical Josephson current. An experimental observation of the predicted effects could lead to a new type of spectroscopy of weak links.

\section{Acknowledgment}

We thank V. Shumeiko and S. Kulinich for fruitfull discussions. Financial support from the Swedish VR (MJ, RS), the Korean WCU program funded by MEST/NFR (R31-2008-000-10057-0) (MJ), the Knowledge Innovation Program (PKIP) of the Chinese Academy of Sciences (KJCX2.YW.W10) (RS), and the National Academy of Science of Ukraine (4/13-N) (IK, AP) is gratefully acknowledged. Partial support for IK was done by the Leading Foreign Research Institute Recruitment Program (2009-00514) of NRF, Korea. IK thanks the Department of Physics at the University of Gothenburg and the Department of Physics and Astronomy at the Seoul National University for hospitality.

1. M. Galperin, M.A. Ratner, and A. Nitzan, J. Phys.: Condens. Matter 19, 103201 (2007).

2. J. Koch and F. von Oppen, Phys. Rev. Lett. 94, 206804 (2005); J. Koch, F. von Oppen, and A.V. Andreev, Phys. Rev. B 74, 205438 (2006).

3. I.V. Krive, A. Palevski, R.I. Shekhter, and M. Jonson, Fiz. Nizk. Temp. 36, 155 (2010) [Low Temp. Phys. 36, 119 (2010)].

4. A.K. Hüttel, M. Poot, B. Witkamp, and H.S.J. van der Zant, New J. Phys. 10, 095003 (2008).

5. P. Utko, R. Ferone, I.V. Krive, R.I. Shekhter, M. Jonson, M. Monthioux, L. Noe, and J. Nygard, Nat. Commun. 1, 37 (2010); Acta Phys. Pol. A 120, 839 (2011).

6. L.Y. Gorelik, A. Isacsson, M.V. Voinova, B. Kasemo, R.I. Shekhter, and M. Jonson, Phys. Rev. Lett. 80, 4526 (1998).

7. R.I. Shekhter, Yu. Galperin, L.Y. Gorelik, A. Isacsson, and M. Jonson, J. Phys.: Condens. Matter 15, R441 (2003).

8. A.Yu. Kasumov, R. Deblock, M. Kociak, B. Reulet, H. Bouchiat, I.I. Khodos, Yu.B. Gorbatov, V.T. Volkov, C. Journet, and M. Burghard, Science 284, 1508 (1999).

9. E. Pallecchi, M. Gaass, D.A. Ryndyk, and C. Strunk, Appl. Phys. Lett. 93, 072501 (2008).
10. A.Yu. Kasumov, M. Kociak, M. Ferrier, R. Deblock, S. Gueron, B. Reulet, I. Khodos, O. Stephan, and H. Bouchiat, Phys. Rev. B 68, 214521 (2003).

11. Recently [12] nanoelectromechanical effects were claimed to be observed in ac Josephson current through a suspended InAs nanowire.

12. A.V. Kretinin, A. Das, and H. Shtrikman, arXiv: 1303.1410 (2013).

13. T. Novotny, A. Rossini, and K. Flensberg, Phys. Rev. B 72, 224502 (2006).

14. A. Zazunov, D. Feinberg, and T. Martin, Phys. Rev. Lett. 97, 196801 (2006).

15. A. Zazunov and R. Eggert, Phys. Rev. B 81, 104508 (2010).

16. J. Sköldberg, T. Löfwander, V.S. Shumeiko, and M. Fogelström, Phys. Rev. Lett. 101, 087002 (2008).

17. A.V. Parafilo, I.V. Krive, R.I. Shekhter, and M. Jonson, Fiz. Nizk. Temp. 38, 348 (2012) [Low Temp. Phys. 38, 273 (2012)].

18. Q.-f. Sun, B.-g. Wang, J. Wang, and T.-h. Lin, Phys. Rev. B 61, 4754 (2000).

19. Y. Meir and N.S. Wingreen, Phys. Rev. Lett. 68, 2512 (1992).

20. S. Maier, T.L. Schmidt, and A. Komnik, Phys. Rev. B 83, 085401 (2011).

21. G.A. Skorobagatko, S.I. Kulinich, I.V. Krive, R.I. Shekhter, and M. Jonson, Fiz. Nizk. Temp. 37, 1295 (2011) [Low Temp. Phys. 37, 1032 (2011)].

22. G.A. Skorobagatko, Phys. Rev. B 85, 075310 (2012).

23. C.W.J. Beenakker and H. van Houten, Single Electron Tunneling and Mesoscopic Devices, Springer, Berlin (1991).

24. G. Wendin and V.S. Shumeiko, Superlatt. Microstr. 20, 569 (1996).

25. I.O. Kulik and A.N. Omelyanchouk, Fiz. Nizk. Temp. 3, 945 (1977) [Sov. J. Low Temp. Phys. 3, 459 (1977)].

26. A.V. Parafilo, I.V. Krive, E.N. Bogachek, and U. Landman, Fiz. Nizk. Temp. 38, 446 (2012) [Low Temp. Phys. 38, 349 (2012)].

27. G. Mahan, Many-Particle Physics, Plenum Press, New York (1990), p. 286.

28. I.S. Gradshtein and I.M. Ryzhik, Tables of Integrals, Series and Products, Academic Press, New York (1980).

29. S. Sapmaz, P. Jarillo-Herrero, Ya.M. Blanter, C. Dekker, and H.S.J. van der Zant, Phys. Rev. Lett. 96, 026801 (2006).

30. H. Park, J. Park, A.K.L. Kim, E.H. Anderson, A.P. Alivisatos, and P.L. McEuen, Nature 407, 58 (2000). 